\title{
Resultados alejados de la resección local transanal para cánceres de recto bajo bien seleccionados
}

\author{
J. Contreras, G. Bannura, A. Barrera, C. Zúñiga, C. Melo y D. Soto \\ Departamento de Cirugía (Centro). Facultad de Medicina. Universidad de Chile. Servicio de Cirugía. Hospital Clínico \\ San Borja-Arriarán. Chile
}

\section{RESUMEN}

Objetivo: evaluar nuestros resultados alejados en términos de recidiva local, rescate y sobrevida libre de enfermedad.

Método: veintiún pacientes con un cáncer de recto bajo fueron sometidos a una resección transanal completa en un periodo de 18 años (1985-2003). La estadificación preoperatoria incluyó hallazgos clínicos y endoscópicos, estudios por imágenes e histopatológicos. Los especímenes resecados correspondieron a 4 tumores T1, 13 T2 y 4 T3.

Los 4 pacientes T1 sólo fueron tratados con cirugía; 6 de los $13 \mathrm{~T} 2$ y todos los T3 recibieron quimiorradioterapia postoperatoria. Ninguno de ellos recibió una cirugía radical secuencial.

Resultados: el seguimiento (media 75 meses) registró 5 casos $(23,8 \%)$ de recidiva total y 4 (19\%) con falla local (3 T2 de los cuales sólo uno había recibido terapia coadyuvante y un T3 también tratado con quimiorradioterapia).

Los cuatro pacientes con recidiva local accedieron a cirugía de rescate, una amputación abdominoperineal en dos casos y a una re-resección local en los otros. Dos de ellos volvieron a recidivar.

La sobrevida a 5 años fue de 95,2\%, y la sobrevida libre de enfermedad en el mismo periodo alcanzó a un 90,4\%. En el periodo de observación hasta agosto de 2006 hay dos pacientes que fallecieron diseminados, y sólo un paciente vivo está recidivado.

Conclusiones: esta experiencia confirma que la resección local transanal para cáncer de recto bajo es una buena alternativa en pacientes adecuadamente seleccionados. En tumores T2, la quimiorradioterapia adyuvante será necesaria; en tumores T3, la cirugía radical de rescate debería ser realizada oportunamente.

Palabras clave: Cáncer de recto. Resección local.

\section{ABSTRACT}

Objective: to evaluate our results about local failure, total recurrence and survival.

Method: twenty-one patients underwent a complete local transanal excision for low rectal cancer in our institution during an 18-year period (1985-2003). Preoperative staging included clinical, endoscopic, CT, EUSR, and histopathological findings; pathological specimens were $4 \mathrm{~T} 1,13 \mathrm{~T} 2$ and 4 T3 tumors.

None of the T1 patients received another treatment; 6 out of 13 T2 and all of T3 cases were treated with chemoradiotherapy. None of the latter underwent radical surgery.

Results: follow-up (median 74 months) proved 23.8\% for total recurrence and $19.0 \%$ for local failure (out of all 3 T2 cases only one received chemoradiotherapy, and one T3 received adjuvant treatment). Five-year survival is $95.2 \%$.

Conclusions: this experience allows us to assert that local excision is a good choice in well-selected low rectal cancer patients; for T2 tumors chemoradiotherapy is necessary; in T3 patients radical surgery must be prompt.

Key words: Rectal cancer. Local excision.

Contreras J, Bannura G, Barrera A, Zúñiga C, Melo C, Soto D. Resultados alejados de la resección local transanal para cánceres de recto bajo bien seleccionados. Rev Esp Enferm Dig 2007; 99: 206-209.

Recibido: 11-10-06.

Aceptado: 27-12-06.

Correspondencia: J. Contreras. Departamento de Cirugía (Centro). Facultad de Medicina. Universidad de Chile. Hospital Clínico San Borja-Arriarán. Chile. e-mail: jcontreras@med.uchile.cl

\section{INTRODUCCIÓN}

El tratamiento convencional de los carcinomas de recto bajo ha sido la resección radical, ya sea una amputación abdominoperineal o una resección anterior ultrabaja. Ambas técnicas presentan índices de morbili- 
dad no despreciables, y secuelas en las funciones urinarias y sexuales en al menos un $15 \%$ de los pacientes. En las resecciones abdominoperineales la colostomía será definitiva. En las resecciones anteriores bajas puede ocurrir una dehiscencia anastomótica hasta en un $15 \%$ de los casos, lo que ha llevado al uso de ileostomías de protección; además, un número significativo de pacientes presentará urgencia defecatoria y suciedad perianal por incontinencia (1-5). Todos estos hechos han incentivado la búsqueda de procedimientos más conservadores como la resección local, la electrocoagulación, la irradiación endocavitaria y la láser terapia en algunos casos seleccionados; estas técnicas, que inicialmente fueron aplicadas en pacientes con un riesgo muy elevado para optar a una cirugía más agresiva, demostraron en su seguimiento tasas de recidiva similares a las de intervenciones radicales (6-8). La resección local endoanal, que ofrece la ventaja de permitir el estudio histopatológico de un espécimen completo para una apropiada estadificación, ha ganado un espacio en el tratamiento de algunos cánceres de recto bajo confinados a la pared, por lo que hoy en día se considera que un 3 a 5\% de los carcinomas de recto puede ser tratado con esta metódica (9-11). Los criterios de selección deben incluir las siguientes condiciones: límite cefálico del tumor no más allá de $7 \mathrm{~cm}$ del margen anal, un tamaño no superior a $5 \mathrm{~cm}$, no adherido a planos profundos, sin evidencia de adenopatías, grado histológico bien o moderadamente diferenciado y una estadificación negativa para enfermedad regional o a distancia $(9,12,13)$. El estudio de anatomía patológica permite determinar el grado de penetración (T), y por tanto qué pacientes recibirán o no quimiorradioterapia coadyuvante, de acuerdo a si la penetración tumoral sobrepasa la capa submucosa y/o existe permeación vascular o linfática. Los tumores que invaden la grasa perirrectal debieran ser sometidos a una resección radical $(6,12,13)$.

El objetivo de este estudio es la evaluación de nuestros resultados alejados en términos de recidiva local, rescate y sobrevida libre de enfermedad.

\section{MATERIAL Y MÉTODO}

En el periodo comprendido entre octubre de 1985 y septiembre de 2003, hemos tratado 209 pacientes portadores de un cáncer de recto medio o inferior; la fecha de corte se debe a la necesidad de contar con un seguimiento de al menos 36 meses. En 21 de ellos (10\%) se extirpó completamente el tumor por vía transanal. Los adenomas malignizados, carcinomas in situ y las resecciones con criterio paliativo fueron excluidos. Se trata de 14 mujeres y 7 hombres, con edades entre 34 y 80 años (media 61). Todos los tumores eran móviles, protruidos en 11 y ulcerados en 10 .
El límite cefálico de todas las lesiones no superaba los $7 \mathrm{~cm}$ desde el margen anal; el tamaño era menor de $3 \mathrm{~cm}$ en 14 , y entre 3 y $5 \mathrm{~cm}$ en 7 . El compromiso parietal se localizaba en la pared posterior en 13, anterior en 4, lateral en 3 y era hemicircunferencial en un caso. Todos los tumores fueron bien o moderadamente diferenciados.

Por medio del examen clínico, al momento del diagnóstico y posteriormente bajo anestesia regional, 9 pacientes fueron catalogados como T1 en 4 y T2 en 5. Sólo 9 pacientes fueron estudiados con endosonografía rectal y 8 con tomografía axial, correspondiendo al periodo 1994 al 2003, cuando los Servicios Públicos de Salud de Chile ya contaban con estos procedimientos diagnósticos. En estos 12 pacientes, la clasificación fue de 3 T1 y 9 T2 .

Quince tenían CEA preoperatorio, menor de $5 \mathrm{ng} / \mathrm{ml}$ en 14 de ellos.

De acuerdo a nuestros protocolos, por tratarse de lesiones tumorales móviles y de una penetración parietal parcial, ningún paciente fue sometido a quimiorradioterapia neoadyuvante, como sí ocurre en tumores fijos y de mayor tamaño.

El seguimiento fue completo hasta agosto de 2006 o en fecha de deceso por causa conocida, con una media de observación de 75 meses. Se determinó la aparición de recidiva local y su relación con el estadio, las conductas adoptadas en los pacientes recidivados y la sobrevida quinquenal.

\section{RESULTADOS}

Se presentaron 2 complicaciones postoperatorias: una hemorragia precoz que fue taponada y una tardía que requirió hospitalización y transfusiones; la herida estaba suturada en el primer caso y abierta en el otro. No se registraron reintervenciones ni hubo mortalidad operatoria.

El estudio anatomopatológico reveló compromiso no más allá de la submucosa (T1) en 4 casos, invasión de muscular propia (T2) en 13, y de la grasa perirrectal (T3) en 4. En estos últimos pacientes, 3 de ellos tenían un estadio uT2NO en la endosonografía, y en 2 la tomografía axial no demostró compromiso de la grasa perirrectal.

A quimiorradioterapia o radioterapia exclusiva accedieron 6 T2 y 3 T3. Ningún T3 fue sometido a cirugía radical en ese momento.

Tres pacientes T2 y un T3 (19\%) recidivaron localmente, cuya evidencia ocurrió a los 13, 14, 23 y 27 meses respectivamente; sólo uno de ellos no había recibido terapia complementaria. En todos se intentó cirugía de rescate, resección abdominoperineal en dos casos y re-resección local en los otros. En dos pacientes se presentó una nueva recidiva.

Hubo 2 casos de metástasis a distancia (pulmón, cerebro); otros 2 sufrieron un segundo cáncer primario (vejiga, próstata).

Veinte pacientes $(95,2 \%)$ estaban vivos a los 5 años, y 16 están actualmente vivos y sin enfermedad manifiesta. 


\section{DISCUSIÓN}

Un $10 \%$ de los carcinomas de recto tratados durante el período de registro accedió a esta técnica, índice muy superior a lo recomendado (4), lo que en parte se debería a la inclusión de tumores mayores de $3 \mathrm{~cm}$ y a la presencia de lesiones ulceradas que cumplían con todas las otras condiciones ya señaladas. Sin embargo, ello conlleva un riesgo mayor de recidiva.

La recidiva local luego de una resección local puede deberse a la persistencia residual microscópica de tejido o células tumorales en el sitio primario, o a la existencia de nódulos linfáticos comprometidos en el mesorrecto, que no serán resecados a través de esta técnica. El riesgo de compromiso metastático está directamente relacionado a la penetración parietal del tumor, en especial de la capa submucosa $(14,15)$. A pesar de la valiosa información que reporta la endosonografía en cuanto a penetración parietal y nódulos linfáticos afectados, su índice de certeza no sobrepasa el 80\% (15,16); así ocurrió con 3 pacientes de esta muestra, que fueron subestadificados.

Los índices de recidiva local informados en las 2 últimas décadas varían de 0 a $44 \%(9,16-19)$. El tamaño y la penetración del tumor, la invasión venosa y de conductos linfáticos, y la morfología ulcerativa constituyen factores de impacto sobre la recidiva local y la sobrevida libre de enfermedad (20). Nuestros 4 casos de recidiva corresponden a tumores ulcerados de $4 \mathrm{~cm}$ de diámetro que al menos penetraban hasta la muscular propia. Otro factor de riesgo elevado es la extirpación del tumor en fragmentos, situación que debe considerarse no curativa y subsidiaria de cirugía radical inmediata (19).

No registramos fallas locales ni enfermedad metastásica en nuestros pacientes T1 tratados con resección local; ello contrasta con resultados desalentadores comunicados en los últimos años, con recidivas de hasta 29\% (21-23).

Análisis retrospectivos en pacientes T2 tratados con terapia coadyuvante informan una recidiva no mayor a $2 \%(8,9,15)$ versus 15 a $20 \%$ en aquellos que sólo fueron resecados $(10,24,25)$; en este estudio sólo 1 de 7 pacientes sin quimiorradioterapia presentó una falla locorregional; pese a ello, nos parece conveniente mantener la conducta de tratamiento combinado (7).

En el caso fortuito de que el estudio histopatológico demuestre compromiso de la grasa perirrectal, el paciente debiera acceder en breve plazo a una cirugía radical, para alcanzar resultados oncológicos similares a los de pacientes resecados inicialmente $(6,10,26)$; sin embargo, recientes estudios demuestran resultados favorables con las terapias coadyuvantes al igual que en los T2 (14). Ambas opciones deben ser discutidas con el paciente, quien debe tener pleno conocimiento de los riesgos de recidiva y las secuelas de uno u otro tratamiento. En nuestra serie hay 2 pacientes que, debidamente informados, rehusaron la posibilidad de una colostomía definitiva y fueron tratados con quimiorradioterapia, con excelentes resultados hasta los 9 y 7 años de seguimiento, respectivamente.
El 95,2\% de sobrevida quinquenal en este estudio es muy superior al de pacientes de estadios similares sometidos a cirugía radical $(27,28)$. Un caso en que aparecieron metástasis pulmonares a los 7 años confirma la necesidad de un seguimiento más prolongado.

\section{CONCLUSIONES}

La resección local transanal de algunos carcinomas de recto bajo puede ser considerada como una opción válida en algunos pacientes, debido a su muy baja morbimortalidad, la preservación de la función esfinteriana, la ausencia de secuelas urinarias y sexuales y resultados oncológicos similares a los pacientes sometidos a cirugía radical.

Sin embargo, estos buenos resultados dependerán del estricto respeto a los protocolos de tratamiento establecidos para la selección y manejo integral de acuerdo al estadio. Los tumores que no invaden más allá de la submucosa podrán ser tratados sólo con resección local; los que progresan hasta la muscular propia requerirán quimiorradioterapia complementaria; aquellos con compromiso de la grasa perirrectal no deberían acceder a esta técnica si el estudio previo predice este estadio; sin embargo, en el caso de ser un hallazgo en el estudio de la pieza resecada, la conveniencia de un tratamiento quirúrgico radical o coadyuvante debería analizarse caso a caso por un comité multidisciplinario.

\section{BIBLIOGRAFÍA}

1. Rothenberger DA, Wong WD. Abdominoperineal resection for adenocarcinoma of the rectum. World J Surg 1992; 16: 478-85.

2. Nesbakken A, Nygaard K, Lunde OC. Bladder and sexual disfunction after mesorectal excision for rectal cancer. Br J Surg 2000; 87: 206-10.

3. Williams NS, Johnston D. The quality of life after rectal excision for low rectal cancer. Br J Surg 1983; 70: 460-2.

4. Karanjia ND, Schache DJ, Heald RJ. Function of the distal rectum after low anterior resection for carcinoma. Br J Surg 1992; 79: 114-6.

5. Astudillo E, Fernández-Cruz L. Técnicas conservadoras del esfínter anal en la cirugía del cáncer de recto. Monografías Clínicas en Oncología. Vol. 8. Cáncer colorrectal. Ediciones Doyma; 1990. p. 115-2.

6. Abel ME, Rosen L, Kodner IJ. Practice parameters for the treatment of rectal carcinoma - Supporting documentation. Dis Col Rectum 1993; 36: 991-1006.

7. Morson BC, Bussey HJ, Samoorian S. Policy of local excision for early cancer of the colorectum. Gut 1977; 18: 1045-50.

8. Whiteway J, Nichols RJ, Morson BC. The role of surgical local excision in the treatment of rectal cancer. Br J Surg 1985; 72: 694-7.

9. Killingback M. Local excision of carcinoma of the rectum: indications. World J Surg 1992; 16: 437-46.

10. Minsky BD. Clinical experience with local excision and postoperative radiation therapy for rectal cancer. Dis Col Rectum 1993; 36: 4059.

11. Taylor RH, Hay JH, Larsson SN. Transanal local excision of selected low rectal cancers. Amer J Surg 1998; 175: 360-3.

12. Bannura G, Contreras J. Resección local curativa en cáncer de recto. Rev Chil Cirugía 1994; 46: 393-8.

13. Nivatvongs S. Surgical management of early rectal cancer. World J Surg 2000; 24: 1052-5.

14. Bleeday R, Breen E, Jassup M. Prospective evaluation of local exci- 
sion for small rectal cancers. Dis Col Rectum 1997; 40: 388-92.

15. García-Aguilar J, Mellgren A, Sirivongs P. Local excision of rectal cancer without adjuvant therapy: A word of caution. Ann Surgery 2000; 231: 345-51.

16. Mellgren A, Sirivongs P, Rothenberger D. Is local excision adequate therapy for early rectal cancer? Dis Col Rectum 2000; 43: 1064-74.

17. Biggers OR, Beart RW, Ilstrup DM. Local excision of rectal cancer. Dis Col Rectum 1986; 29: 374-7.

18. Masaki T, Sugiyama M, Atomi H. The indication of local excision for T2 rectal carcinomas. Amer J Surg 2001; 181: 133-7.

19. Gopaul D, Belliveau P, Vuong T. Outcome of local excision of rectal carcinoma. Dis Col Rectum 2004; 47: 1780-8.

20. Hager Th, Gall FP, Harmanek P. Local excision of cancer of the rectum. Dis Col Rectum 1983; 26: 149-51.

21. Sengupta S, Tjandra JJ. Local excision of rectal cancer: what is the evidence? Dis Col Rectum 2001; 44: 1345-61.

22. Endreseth B, Myrvold H, Romundstad P. Transanal excision vs. major surgery for T1 rectal cancer. Dis Col Rectum 2005; 48: 1380-8.
23. Madbouly K, Remzi F, Erkek B. Recurrence after transanal excision of T1 rectal cancer: should we be concerned? Dis Col Rectum 2005; 48: 711-21.

24. Contreras J. Resección endoanal curativa en cáncer de recto: resultados alejados y evaluación de protocolo. Rev Chil Cirugía 2002; 54: 182-8.

25. Minsky BD, Enker WE, Cohen AM. Clinicopathological features in rectal cancer treated by local excision and postoperative radiation therapy. Radiat Med 1995; 13: 235-41.

26. Hahnloser D, Wolff B, Larson D. Inmediate radical resection after local excision of rectal cancer: an oncologic compromise? Dis Col Rectum 2005; 48: 429-37.

27. McDermott FT, Hughes ES, Pihl E. Local recurrence after potentially curative resection for rectal cancer in a series of 1008 patients. Br J Surg 1985; 72: 34-7.

28. Bannura G, Contreras J, Cumsille MA. Resultados del tratamiento quirúrgico del cáncer de recto: análisis de 180 pacientes. Rev Chil Cirugía 1998; 50: 646-54. 